\title{
QUASI-ANALYTICITY AND SEMIGROUPS
}

\author{
BY J. W. NEUBERGER ${ }^{1}$
}

ABSTRACT. Four problems and their interrelationships are considered. These problems concern (1) quasi-analyticity conditions in terms of finite differences, (2) quasi-analyticity conditions for one-parameter semigroups of linear transformations, (3) generation (in the sense of S. Lie) of one-parameter semigroups of nonlinear transformations and (4) quasi-analyticity conditions for one-parameter semigroups of nonlinear transformations. The quasi-analyticity conditions in (2) and (4) are in terms of the degree of approximation of the identity by a semigroup. In connection with (3) an infinitesimal generator and a corresponding exponential formula are obtained without assuming differentiability.

1. This work deals with quasi-analyticity, with linear and nonlinear one-parameter semigroups of transformations and with certain relationships between these subjects. If $J$ is a connected set of real numbers, then a collection $G$ of functions with common domain $J$ is said to be quasianalytic provided no two members of $G$ agree on an open subset of $J$.

Suppose $H$ is a Banach space. If $C$ is a subset of $H$, a semigroup on $C$ is a function $T$ with domain $[0, \infty)$ such that

(1) If $\lambda \geqq 0$, then $T(\lambda)$ is a transformation from $C$ to $C$, and

(2) If $s, t \geqq 0$, then $T(t) T(s)=T(t+s)$ and $T(0)$ is the identity transformation on $C$.

If $p$ is in $C$, then $g_{p}$ denotes the function from $[0, \infty)$ to $C$ such that $g_{p}(\lambda)=T(\lambda) p$ for all $\lambda \geqq 0\left(g_{p}\right.$ is called a trajectory of $\left.T\right)$. If $p$ is in $C$ and $f$ is in $H^{*}$, then the composition $f g_{p}$ is denoted by $z_{p, f}$ and is called a functional of a trajectory of $T$. If $r \geqq 0$, then $T$ is called $r$-quasi-analytic provided no two continuous functionals of trajectories of $T$ agree on an open subset of $(r / 2, \infty)$ unless they agree on all of $(r / 2, \infty)$. If $T$ is $r$-quasianalytic and $r=0$, then $T$ will be called quasi-analytic. If $g_{p}$ is continuous for all $p$ in $C$, then $T$ is called strongly continuous.

Quasi-analytic collections of real-valued functions have been studied by S. Bernstein, Carleman, Denjoy, Wiener, Beurling, de la Vallée Poussin, Mandelbrojt and others. Descriptions of Bernstein's work may be found in [3] and [37]. The reader is also referred to Carleman's book [6], to

\footnotetext{
${ }^{1}$ A slightly expanded version of an address delivered to the Auburn meeting of the Society by invitation of the Committee to Select Hour Speakers for Southeastern Sectional Meetings, November 19, 1971; received by the editors March 27, 1972. Work supported in part by a grant from the National Science Foundation (1969-71) and by a fellowship from the Alfred P. Sloan Foundation (1967-69).

AMS 1970 subject classifications. Primary 26A93, 47D05, 47H15; Secondary 60J25, 42A44.

Key words and phrases. Quasi-analyticity, one-parameter semigroup of transformations, trajectory, Markov semigroup, finite differences, Fourier transform, generator, approximation of identity, exponential formula.
} 
the book of Paley and Wiener [33], Mandelbrojt's books [18], [19], de la Vallée Poussin's paper [38] and Beurling's lecture notes [1]. There seem to be few established relationships between the above works and the quasi-analyticity theorems of the present work although such relationships have been sought. More will be said on this point later.

Following are some examples of semigroups. A semigroup $T$ is said to be linear if $T(\lambda)$ is a linear transformation for all $\lambda \geqq 0$. Otherwise $T$ is called nonlinear.

EXAMPLE 1. $H=C=E_{2}$,

$$
T(\lambda)=\left(\begin{array}{rr}
\cos \lambda & \sin \lambda \\
-\sin \lambda & \cos \lambda
\end{array}\right), \quad \lambda \geqq 0 .
$$

EXAMPLE 2. $H=C=l_{1}$,

$$
T(\lambda)\left(\begin{array}{c}
x_{1} \\
x_{2} \\
\vdots
\end{array}\right)=\left(\begin{array}{c}
x_{1} e^{-\lambda} \\
x_{2} e^{-2 \lambda} \\
\vdots
\end{array}\right), \quad \lambda \leqq 0,\left(\begin{array}{c}
x_{1} \\
x_{2} \\
\vdots
\end{array}\right) \text { in } l_{1} .
$$

EXAMPLe 3. $H=R, C=[0,1], T(\lambda) p=p^{\left(e^{\lambda}\right)}, \lambda \geqq 0, p$ in $[0,1]$.

EXAMPLE 4. $H=R, C=[-1,1]$,

$$
T(\lambda) x \begin{cases}=x & \text { if }-1 \leqq x \leqq 0 \\ =0 & \text { if } 0 \leqq x \leqq \lambda, 0 \leqq x \leqq 1, \lambda \geqq 0, \\ =x-\lambda & \text { if } 1 \geqq x \geqq \lambda .\end{cases}
$$

Example 5. $H=R, C=[0,1], T(\lambda) x=x /(1+x \lambda), 0 \leqq x \leqq 1, \lambda \geqq 0$.

EXAMPLE 6. $H=C=l_{2},\left\{n_{i}\right\}_{i=1}^{\infty}$ an increasing sequence of positive integers,

$$
T(\lambda)\left(\begin{array}{c}
x_{1} \\
y_{1} \\
x_{2} \\
y_{2} \\
\vdots
\end{array}\right)=\left(\begin{array}{c}
x_{1} \cos n_{1} \lambda+y_{1} \sin n_{1} \lambda \\
-x_{1} \sin n_{1} \lambda+y_{1} \cos n_{1} \lambda \\
\vdots
\end{array}\right),\left(\begin{array}{c}
x_{1} \\
y_{1} \\
x_{2} \\
y_{2} \\
\vdots
\end{array}\right) \text { in } l_{2}, \lambda \geqq 0 .
$$

EXAMPLE 7. $H=C=$ space (under sup norm) of continuous realvalued functions $f$ on $(-\infty, 1]$ such that $f(x)=0$ if $x \leqq 0$. $[T(\lambda) f](x)$ $=f(x-\lambda), x \leqq 1, \lambda \geqq 0, f$ in $H$.

EXAMPLe 8 (G. F. WeBb). $H=C=C_{[0,1]} \cdot F(x)=x$ if $x \geqq 0,2 x$ if $x<0 .[T(\lambda) f](x)=F\left(\lambda+F^{-1}(f(x))\right), 0 \leqq x \leqq 1, \lambda \geqq 0, f$ in $C_{[0,1]}$.

EXAMPLE .9. $H=R, a \neq b, a, b>0, C=[-1,1], T(\lambda) x=e^{-a \lambda}$ if $0 \leqq x \leqq 1, e^{-b \lambda}$ if $-1 \leqq x<0, \lambda \geqq 0$.

2. Some long-known facts about linear semigroups are summarized in the following (cf. [11], [42]). 
THEOREM 1. Suppose $T$ is a strongly continuous semigroup of bounded linear transformations. Then,

(1) $\lim _{\lambda \rightarrow 0}(1 / \lambda)(T(\lambda)-I) p$ exists for all $p$ in some dense subset of $H$ $(B$ denotes the transformation with domain all such $p$ and $B p$ is this limit for $p$ in $D_{B}$ ).

(2) There is $\lambda_{0}>0$ such that $(I-\lambda B)^{-1}$ exists and is a bounded linear transformation from $H$ to $H$ if $0 \leqq \lambda \leqq \lambda_{0}$.

(3) $\lim _{n \rightarrow \infty}(I-(\lambda / n) B)^{-n} p=T(\lambda) p$ for all $\lambda \geqq 0$ and all $p$ in $H$.

This theorem is a remarkable instance of algebraic and topological conditions leading to the existence of differentiability. In terms of this differentiability, and 'infinitesimal generator' $B$ of $T$ is defined. The semigroup $T$ is then recovered from $B$ by means of the exponential formula (3).

In recent years there has been considerable success in generalizing Theorem 1 to the case of $T$ nonlinear. If $H$ is a Hilbert space, $C$ a convex subset of $H$ and $T$ nonexpansive $(T(\lambda)$ has Lipschitz norm not exceeding 1 for all $\lambda \geqq 0$ ) then the theory is fairly complete. A recent paper of Brezis and Pazy [4] contains a good summary. More will be said about this theory later when another approach to the nonlinear theory is mentioned.

Two quasi-analyticity theorems and one analyticity theorem for linear semigroups follow.

THEOREM 2. If $T$ is a strongly continuous semigroup of bounded linear transformations on $H$ and $\lim _{\inf _{\lambda \rightarrow 0}}|T(\lambda)-I|<2$, then $T$ is quasianalytic.

THEOREM 3. Suppose $T$ is a semigroup of bounded linear transformations on $H$ and

(1) If $\lambda_{0}>0$, then there is $M$ so that $|T(\lambda)| \leqq M$ for all $\lambda$ in $\left[0, \lambda_{0}\right]$.

(2) There is a sequence $\{\delta(q)\}_{q=1}^{\infty}$ of positive numbers converging to $0, \varepsilon, \delta>0$ and $r \geqq 0$ so that

$$
\sup _{r \leqq n \delta(q) \leqq r+\delta}\left|(T(\delta(q))-I)^{n}\right|^{1 / n} \leqq 2-\varepsilon, \quad q=1,2, \ldots .
$$

Then, $T$ is $r$-quasi-analytic.

THEOREM 4. Suppose that $T$ is a strongly continuous semigroup of bounded linear transformations from $H$ to $H$ and $\limsup _{\lambda \rightarrow 0}|T(\lambda)-I|<2$. Then, each functional of a trajectory of $T$ is real-analytic on $(0, \infty)$. Moreover, $B(T(\lambda))$ is bounded for all $\lambda>0$ where $B$ is the infinitesimal generator of $T$.

Theorem 4 is found (essentially) in [25]. It can now be obtained as a special case of a result of Beurling [2] or of Kato [14]. Theorem 2 is found in [26] and [29]. Theorem 3 is found in [29]. Earlier results in this direction are found in [15] and [22]. 
Theorems 2 and 4 apply to Markov semigroups. Following D. G. Kendall $\left[15\right.$, pp. 11, 19-21] suppose each of $p_{i j}, i, j=0,1,2, \ldots$, is a continuous function on $[0, \infty), p_{i j}(u+v)=\sum_{k=0}^{\infty} p_{i k}(u) p_{k j}(v), p_{i j}(t) \geqq 0$, $\sum_{j=0}^{\infty} P_{i j}(t)=1, \lim _{t \rightarrow 0} p_{i j}(t)=\delta_{i j}, i, j=0,1,2, \ldots$. These are the transition probability functions for a Markov process. From these one defines a semigroup $T$ on $l_{1}$ :

$$
(T(t) x)_{j}=\sum_{i=0}^{\infty} x_{i} p_{i j}(t), \quad t \geqq 0, j=0,1,2, \ldots, x \text { in } l_{1} .
$$

As Kendall notes, $|T(t)-I|=2(1-g(t))$ where $g(t)=\inf _{i=0,1,2, \ldots} p_{i i}(t)$, $t \geqq 0$. Denote $\lim \sup _{t \rightarrow 0} g(t)$ by $\Gamma$ and $\lim \inf _{t \rightarrow 0} g(t)$ by $\gamma$. Then the hypothesis of Theorem 2 is satisfied if and only if $\Gamma>0$ and the hypothesis of Theorem 4 is satisfied if and only if $\gamma>0$.

3. Theorems 2 and 3 follow from some results which relate quasianalyticity with the study of finite differences. These results are noted here in some detail.

If each of $u$ and $\delta$ is a number, $n$ a positive integer and $f$ a real or complex valued function whose domain includes $[u, u+n \delta]$, then $\Delta_{f}(n ; u, \delta)$ denotes

$$
\sum_{k=0}^{n}\left(\begin{array}{l}
n \\
k
\end{array}\right)(-1)^{n-k} f(u+k \delta),
$$

the $n$th difference of $f$ from $u$ to $u+n \delta$. Observe that if $|f(x)| \leqq M$ for all $x$ in $[u, u+n \delta]$, then one has the crude inequalities $\left|\Delta_{f}(n ; u, \delta)\right| \leqq M 2^{n}$ and $\left|\Delta_{f}(n ; u, \delta)\right|^{1 / n} \leqq 2 M^{1 / n}$ since $\sum_{k=0}^{n}\left(\begin{array}{l}n \\ k\end{array}\right)=2^{n}$. Recall that if $f$ is $C^{(n)}$ in some open set containing $u$, then $\Delta_{f}(n ; u, \delta) / \delta^{n} \rightarrow f^{(n)}(u)$ as $\delta \rightarrow 0$. The next two examples are intended to introduce Theorem 5 .

EXAmPle 10. Suppose

$$
f(t)= \begin{cases}0, & \text { if } 0 \leqq t<1 / 2 \\ t-1 / 2, & \text { if } 1 / 2<t \leqq 1\end{cases}
$$

Some computation gives

$$
\left|\Delta_{f}(2 n ; 0,1 / 2 n)\right|=(1 / n)\left(\begin{array}{c}
2 n-2 \\
n-1
\end{array}\right)
$$

and so $\left|\Delta_{f}(2 n ; 0,1 / 2 n)\right|^{1 / 2 n} \rightarrow 2$ as $n \rightarrow \infty$.

EXAMPLE 11. Suppose

$$
f(t)= \begin{cases}0, & \text { if } 0 \leqq t \leqq 1 / 2 \\ (t-1 / 2)^{2}, & \text { if } 1 / 2<t \leqq 1\end{cases}
$$

Then $\left|\Delta_{f}(2 n ; 0,1 / 2 n)\right|=0, n=2,3,4, \ldots$ but 
$\left|\Delta_{f}(2 n-1 ; 0,1 / 2 n)\right|=(4 n-1) n^{-2}(n-1)^{-1}\left(\begin{array}{c}2 n-4 \\ n-2\end{array}\right), \quad n=2,3,4, \ldots$

So, if $y<1$, then $\left(\sup _{y<q / 2 n<1}\left|\Delta_{f}(q ; 0,1 / 2 n)\right|\right)^{1 / 2 n} \rightarrow 2$ as $n \rightarrow \infty$. For these examples, the crude upper bound mentioned above is, in a sense, attained. The essence of Theorem 5 is that this situation is typical for a wide class of functions.

If $x \geqq 0$, then $[x]$ denotes the largest integer which does not exceed $x$.

THEOREM 5. Suppose $f$ is a continuous function with domain $[0,1]$ so that $f(t)=0$ if $0 \leqq t \leqq 1 / 2$ and, if $z>1 / 2$, then there is $t$ in $(1 / 2, z)$ such that $f(t) \neq 0$. If $y<1$ and $\{\delta(q)\}_{q=1}^{\infty}$ is a sequence of positive numbers converging to 0 , then

$$
\lim _{q \rightarrow \infty}\left(\sup _{y \leqq n \delta(q) \leqq 1}\left|\Delta_{f}(n ; 0, \delta(q))\right|\right)^{1 /[1 / \delta(q)]}=2 .
$$

No constructive argument for Theorem 5 is known. It is easy to see that

$$
\limsup _{q \rightarrow \infty}\left(\sup _{y \leqq n \delta(q) \leqq 1}\left|\Delta_{f}(n ; 0, \delta(q))\right|\right)^{1 /[1 / \delta(q)]} \leqq 2,
$$

assuming that lim $\sup _{q \rightarrow \infty}()<2$ leads by a long argument to a contradiction (see [21] and also [29]). Any information concerning just how the limit in Theorem 5 is achieved would almost certainly lead to substantial improvements in Theorems 2 and 3.

A key lemma in the proof of Theorem 5 is the following inequality (a slight improvement of Lemma 4 of [21]):

Suppose that each of $u, j, s$ and $t$ is a positive integer, $u \leqq t \leqq s$, each of $a_{u}, \ldots, a_{s+j}$ is a number and $A_{v}=\sum_{i=u}^{v}\left(\begin{array}{c}v \\ i\end{array}\right)(-1)^{v-i} a_{i}, v=t, s+1, \ldots$, $s+j$. Denote $\left|A_{v}\right| /\left(\begin{array}{l}v \\ u\end{array}\right)$ by $F_{v}, v=t, s+1, \ldots, s+j, \max \left\{\left|a_{u}\right|, \ldots,\left|a_{s+j}\right|\right\}$ by $M$ and $\max \left\{F_{s+1}, \ldots, F_{s+j}\right\}$ by $B$. Then,

$$
\begin{gathered}
F_{t} \leqq M \sum_{i=u+j}^{s+j}\left(\left(\begin{array}{c}
s+j \\
i
\end{array}\right) /\left(\begin{array}{c}
s+j \\
u
\end{array}\right)\right)\left(\begin{array}{c}
i-u \\
j
\end{array}\right) \\
\cdot((s-t+j) \cdots(s-t+1)) /((s-u+j) \cdots(s-u+1)) \\
+B\left(\begin{array}{c}
2(s-t+j)-1 \\
j-1
\end{array}\right) .
\end{gathered}
$$

Suppose $J$ is a connected set of numbers and $r \geqq 0$ such that $J$ contains an interval of length greater than $r$. Then $J_{r}$ denotes $\{x:[x-r / 2$, 
$x+r / 2] \subset J\}$ and, if $G$ is a collection of functions with domain $J$, then $G_{r}$ denotes the collection of all restrictions of members of $G$ to $J_{r}$.

THEOREM 6. Suppose $r$ and $J$ are as above and suppose furthermore that $G$ is a collection of continuous functions on $J$ such that if $f$ is in $G$, then there are $M, \varepsilon, \delta>0$ such that

$$
\left|\Delta_{f}(n ; u, \delta(q))\right| \leqq M(2-\varepsilon)^{n}
$$

provided $n, q$ are positive integers, $u, u+n \delta(q)$ are in $J$ and $r \leqq n \delta(q)$ $\leqq r+\delta$. Then $G_{r}$ is a quasi-analytic collection.

Theorem 6 appears in [29] and in a sense is an improvement of Theorem B of [21]. Denote $\{\delta(q)\}_{q=1}^{\infty}$ by $\sigma$ and denote by $G(\sigma, J)$ the set of all continuous real-valued functions $f$ with domain $J$ such that if $x$ is in $J$, then there is an open interval $J^{\prime}$ containing $x$ and $M, \varepsilon>0$ so that if $u, v$ are in $J^{\prime} \cap J$ and $v=u+n \delta(q)$ for some integers $q$ and $n$, then

$$
\left|\Delta_{f}(n ; u, \delta(q))\right| \leqq M(2-\varepsilon)^{n} .
$$

It follows from Theorem 6 that $G(\sigma, J)$ is a quasi-analytic collection.

A number of questions about such quasi-analytic collections are unanswered:

(1) Is $G(\sigma, J)$ a ring (using pointwise multiplication)?

(2) If $f$ is in $G(\sigma, J)$ and $f$ is known on a subinterval $J^{\prime}$ of $J$, how can $f$ be calculated on all of $J$ ?

(3) Is it true that if $f$ is a continuous function on $J$, then there exist sequences $\sigma^{\prime}$ and $\sigma^{\prime \prime}$ of positive numbers converging to 0 so that $f=f_{1}+f_{2}, f_{1}$ is in $G\left(\sigma^{\prime}, J\right)$ and $f_{2}$ is in $G\left(\sigma^{\prime \prime}, J\right)$ ?

For certain quasi-analytic collections in the literature, a problem similar to (2) has been solved (cf. [6]). For Bernstein's quasi-analytic collections (defined in terms of best approximation by polynomials) the question similar to (3) has been solved in the affirmative (cf. [37]). Question (1) is due to P. Porcelli (private communication).

4. The question arises as to whether $G(\sigma, J)$ contains other than realanalytic functions. For certain choices of $J$ and $\sigma$ the answer is yes. Members of $G(\sigma, J)$, for certain $J$ and $\sigma$, have been found which are not even twice continuously differentiable. Such functions have been exhibited by means of Fourier transforms and series. A relationship between Fourier analysis and the study of quasi-analyticity by means of finite differences is given by the following:

Suppose $\alpha$ is a complex-valued function which is bounded variation on all of $R$. Suppose also that

$$
f(x)=\operatorname{Re} \int_{-\infty}^{\infty} e^{i v x} d \alpha(v) \text { for all } x \text { in } R .
$$


Then

$$
\Delta_{f}(n ; u, \delta)=\operatorname{Re} \int_{-\infty}^{\infty}\left(e^{i \delta v}-1\right)^{n} e^{i v u} d \alpha(v)
$$

and hence

$$
\left|\Delta_{f}(n ; u, \delta)\right| \leqq \int_{-\infty}^{\infty}\left|e^{i \delta v}-1\right|^{n}|d \alpha(v)|=2^{n} \int_{-\infty}^{\infty}|\sin (\delta v / 2)|^{n}|d \alpha(v)|
$$

for all $u, \delta$ in $R$ and all positive integers $n$.

Roughly, if $\alpha$ is constant on intervals of sufficient length about numbers $v$ so that $|\sin (\delta v / 2)|=1$, then for some $\varepsilon>0,\left|\Delta_{f}(n ; u, \delta)\right|$ will be bounded by $(2-\varepsilon)^{n} \int_{-\infty}^{\infty}|d \alpha(v)|$ for all $u$ in $R, n=1,2, \ldots$. An example illustrates this.

Take $\alpha$ to be the nondecreasing step function on $R$ so that $\alpha$ has jumps $3^{-3 p / 2}$ at $3^{p}, p=1,2, \ldots$, and $\alpha$ has no other discontinuities. Choose $\sigma=\left\{2 \pi / 3^{q}\right\}_{q=1}^{\infty}$ and

$$
f(x)=\operatorname{Re} \int_{-\infty}^{\infty} e^{i v x} d \alpha(v)=\sum_{p=1}^{\infty}\left(\cos \left(x 3^{p}\right)\right) / 3^{3 p / 2}
$$

for all $x$ in $R$. Then,

$$
\begin{gathered}
\left|\Delta_{f}\left(n ; u, 2 \pi / 3^{q}\right)\right| \leqq 2^{n} \int_{-\infty}^{\infty}\left|\sin \left(2 \pi v /\left(2 \cdot 3^{q}\right)\right)\right|^{n}|d \alpha(v)| \\
=2^{n} \sum_{p=1}^{\infty}\left|\sin \left(\pi 3^{p-q}\right)\right|^{n} / 3^{3 p / 2}
\end{gathered}
$$

But there is $\varepsilon>0$ so that if $r$ is an integer-positive, negative or zerothen $\left|2 \sin \pi 3^{r}\right| \leqq 2-\varepsilon$. Hence

$$
\left|\Delta_{f}\left(n ; u, 2 \pi / 3^{q}\right)\right| \leqq(2-\varepsilon)^{n} \sum_{p=1}^{\infty} 3^{-3 p / 2}, \quad n, q=1,2, \ldots
$$

Hence the restriction of $f$ to $[0,7]$ is in $G(\sigma,[0,7])$. But $f$ (and hence its restriction to $[0,7])$ is not $C^{(2)}$ (cf. [36, p. 53]).

Suppose $G$ is the collection of all functions $h$ on $[0,7]$ for which there exists a real number sequence $\left\{a_{p}\right\}_{p=1}^{\infty}$ such that $\sum_{p=1}^{\infty}\left|a_{p}\right|$ converges and $f(x)=\sum_{p=1}^{\infty} a_{p} \cos 3^{p} x$ for all $x$ in $[0,7]$. Then, as above, it may be shown by means of Theorem 6 that $G$ is a quasi-analytic collection.

Much more general quasi-analytic collections than the one indicated above can be constructed using Fourier series and integrals (cf. Theorem 12 of [24]). This last mentioned result can be improved substantially starting with Theorem 5 of the present paper instead of Theorem 12 of [24]. Many of the collections of Fourier series or integrals which can be shown to be quasi-analytic using Theorem 6 can also be shown to be quasi- 
analytic using Theorem IV and Corollary 4.1 (Lecture 3) of [1]. In this connection see also [33], [38], [18]. It is remarked that Mandelbrojt in the preface to [18] credits de la Vallée Poussin and S. Bernstein with introducing Fourier series into the study of quasi-analyticity.

Consider the following result of Beurling [2] (see also [1], [25]).

THEOREM 7. Suppose $f$ is a continuous real-valued function on $[-4,4]$ and that for some $\rho$ in $[3 / 2,2),\left|\Delta_{f}(n ; u,(v-u) / n)\right| \leqq \rho^{n}$ if $u$ and $v$ are in $[-4,4]$, $n=1,2, \ldots$. Then $f$ can be extended analytically to the rhombus with vertices at $\pm 4, \pm 4 i k \alpha^{2}$ where $\alpha=(2-\rho) / 4$ and $k$ is a number not depending on $f$ or $\rho$.

Beurling's argument is based on Fourier analysis. The question is raised as to whether Theorem 6 has a Fourier-analytic proof. The same question can be raised concerning Theorems 2 and 3 . Theorem 4 is already a special case of Theorem IV of [2] whose argument may be described as Fourieranalytic.

D. Williams (private communication) raised the question as to whether Theorem 5 has an argument based on semigroup theory. It would also be interesting to have a Fourier-analytic argument for Theorem 5 .

The quasi-analytic collections considered in [21] were defined in terms of conditions on the finite differences of members of the collection. Theorem 7 implies that certain of the quasi-analytic collections in [21] contain nothing but analytic functions.

D. G. Kendall in [15] defined quasi-analytic collections by means of conditions on some finite differences of its members. In [24] and [29] (and Theorem 6) Kendall's idea was extended to get quasi-analytic collections which contain nonanalytic members.

5. For purposes of comparison one is reminded that for $T$ a semigroup of bounded linear transformations, $\lim _{\lambda \rightarrow 0}|T(\lambda)-I|=0$ if and only if $B$, its generator in the sense of Theorem 1 , is bounded.

In Example 2, $|T(\lambda)-I|=1$ for all $\lambda>0$ and so $T$ satisfies the hypothesis of Theorem 4. The generator $B$ of $T$ is given by

$$
B\left(\begin{array}{c}
x_{1} \\
x_{2} \\
\vdots
\end{array}\right)=\left(\begin{array}{c}
-x_{1} \\
-2 x_{2} \\
\vdots
\end{array}\right)
$$

provided both points involved are in $l_{1}$.

In Example 6, if $n_{k}=k, k=1,2, \ldots$, then $|T(\lambda)-I|=2$ for all $\lambda>0$. Hence $T$ does not satisfy the hypotheses of either Theorem 2 or Theorem 4 . That it does not satisfy the hypothesis of Theorem 4 can be argued another way. Each $T(\lambda), \lambda \geqq 0$, has a bounded inverse defined on all of $l_{2}$. If the hypothesis of Theorem 4 were satisfied, then $B T(\lambda)$ would be bounded 
for all $\lambda>0$. But then $B(T(\lambda)(T(\lambda)))^{-1}$ would be bounded too, a contradiction since $B$ is unbounded:

$$
B\left(\begin{array}{c}
x_{1} \\
y_{1} \\
x_{2} \\
y_{2} \\
\vdots
\end{array}\right)=\left(\begin{array}{c}
n_{1} y_{1} \\
-n_{1} x_{1} \\
n_{2} y_{2} \\
-n_{2} x_{2} \\
\vdots
\end{array}\right)
$$

provided both points involved are in $l_{2}$.

However, if $n_{k}=3^{k}, k=1,2, \ldots$, then $\liminf _{\lambda \rightarrow 0}|T(\lambda)-I|<2$, since if $q$ is a positive integer, then

$$
\begin{aligned}
\left|T\left(2 \pi / 3^{q}\right)-I\right| & =\sup _{k=1,2, \ldots}\left|\exp \left(2 \pi i 3^{k} / 3^{q}\right)-1\right| \leqq \sup _{r=0, \pm 1, \ldots}\left|\exp \left(2 \pi i 3^{r}\right)-1\right| \\
& =|\exp (2 \pi i / 3)-1|<2 .
\end{aligned}
$$

In Example 7 (see [29]) it may be seen that if $n$ is a positive integer, $\delta>0$ and $n \delta \geqq 3$, then

$$
\left|(T(\delta)-I)^{n}\right|^{1 / n} \leqq 2^{1 / n}\left(\begin{array}{c}
n \\
{[1 / \delta]}
\end{array}\right)^{1 / n} \rightarrow 1 \quad \text { as } n \rightarrow \infty,
$$

from which it follows that $\lim _{n \rightarrow \infty}\left|(T(\delta)-I)^{n}\right|^{1 / n} \leqq 1$ and so the hypothesis of Theorem 3 is satisfied. This may be contrasted with the fact that $\lim _{\delta \rightarrow 0}|T(\delta)-I|=2$. It is mentioned that the generator $B$ for this example is given by $B f=-f^{\prime}$ if $f$ and $f^{\prime}$ are in $H$.

Observe that if $T$ is a semigroup of bounded linear transformations on $H, p$ is in $H, f$ is in $H^{*}, u, \delta \geqq 0$, then

$$
\left|\Delta_{z p, f}(n ; u, \delta)\right| \leqq|f|\|p\||T(u)|\left|(T(\delta)-I)^{n}\right| .
$$

This gives a relationship between sizes of differences of functionals of trajectories of $T$ and the degree of approximation of the identity by the semigroup $T$. This inequality provides a basis for a proof of Theorems 2 and 3 by means of Theorem 6 . It may also be used as part of a proof of Theorem 4 by means of Theorem 7 .

Recent work of Pazy [32] uses a weaker condition than in Theorem 4 and still concludes that $B T(\lambda)$ is bounded for all $\lambda>0$ but that it need not be true that each functional of a trajectory of $T$ is analytic. Other references concerning relationships between properties of semigroups and the nature of the approximation of the identity by the semigroup are [5], [41], [9] and $[11, \S 10.7]$.

6. The following theorem for nonlinear semigroups is suggested by two developments. One is Theorem 1 and the other is found in certain work of Sophus Lie (cf. [32], [12]). For a certain kind of one-parameter 
group of nonlinear transformations on a finite dimensional space $H_{1}$, Lie considered a representation as a group of linear transformations on a space $Q_{1}$ of real-valued functions on $H_{1}$. Lie seems to assume differentiability (even analyticity) of the nonlinear group as well as analyticity of the members of $Q_{1}$. In the present theorem, one has semigroups instead of groups, Banach spaces instead of finite dimensional spaces and no differentiability is assumed.

Denote by $C$ a bounded subset of the Banach space $H$. Denote by $K$ a Banach space. Denote by $T$ a nonlinear semigroup on $C$ and by $Q_{0}$ the set of all bounded functions $f$ from $C$ to $K$ such that the composition $f g_{p}$ is continuous for all $p$ in $C$. A subspace $Q$ of $Q_{0}$ may have the following property $\left(Q_{0}\right.$ itself does):

(*) $Q$ is closed under taking Laplace transforms, i.e., if $f$ is in $Q, \lambda>0$ and

$$
h(x)=(1 / \lambda) \int_{0}^{\infty} d u e^{-u / \lambda} f(T(u) x)
$$

for all $x$ in $C$, then $h$ is in $Q$.

THEOREM 8. If $Q$ is a subspace (not necessarily complete) satisfying (*) and $A$ is the set of all $(f, h)$ in $Q \times Q$ such that

$$
\lim _{\lambda \rightarrow 0}(1 / \lambda)(f(T(\lambda) x)-f(x))=h(x)
$$

for all $x$ in $C$, then $(I-\lambda A)^{-1}$ exists, has domain all of $Q,\left|(I-\lambda A)^{-1}\right| \leqq 1$, and

$$
\lim _{n \rightarrow \infty}\left((I-(\lambda / n) A)^{-n} f\right)(x)=f(T(\lambda) x)
$$

for all $f$ in $Q, x$ in $C, \lambda \geqq 0$.

See [28] for a proof. See [27] for a related development which gives more information about $A$. Note that if $\lim _{\lambda \rightarrow 0}(1 / \lambda)(T(\lambda) x-x) \equiv B x$ exists and the member $f$ of $Q$ is Fréchet differentiable, then $(A f)(x)$ $=f^{\prime}(x) B x$. This illustrates a close relationship between the two kinds of generators.

The question is raised: Under what conditions on $A$ does it arise from a semigroup as in Theorem 8?

As a first step in trying to develop a theory of differential equations for nonlinear semigroups, one tries to differentiate a semigroup at 0 and then reconstruct the semigroup from the generator by means of an exponential formula. This step is, for strongly continuous linear semigroups, illustrated by Theorem 1. For nonlinear semigroups this has been accomplished for nonexpansive strongly continuous semigroups on Hilbert space (cf. [16], [13], [10], [4]) but not, for example, on $C_{[0,1]}$. See, for example, 
[7], [8], [20], [35], [40], [30], [23] for developments for more general spaces. In Webb's example from [39] (Example 8 here) on $C_{[0,1]}$, $\lim _{\lambda \rightarrow 0}(1 / \lambda)(T(\lambda) p-p) \equiv B p$ exists (even in the pointwise sense) and is a member of $C_{[0,1]}$ only for $p$ a nonnegative or a negative member of $C_{[0,1]}$ $\left((B p)(x)=1\right.$ if $p \geqq 0,=2$ if $p<0, p$ in $C_{[0,1]}, x$ in $\left.[0,1]\right)$.

Theorem 8 can be interpreted to give a modified sense of differentiability for semigroups on $C_{[0,1]}$ (as well as on much more general spaces) so that a generator, from which the semigroup is recoverable, can be defined in terms of this differentiability. Take $C$ to be a bounded subset of $C_{[0,1]}$ and $T$ a semigroup on $C$ such that if $p$ is in $C, x$ is in $[0,1]$ and $z(t)=(T(t) p)(x)$ for all $t \geqq 0$, then $z$ is continuous (this is a weaker assumption than that of strong continuity of $T$ ). Take $K=R$, the real numbers, and take $Q_{0}$ as above for this choice of $C, T$ and $K$. If $x$ is in $[0,1]$ denote by $f_{x}$ the member of $Q_{0}$ such that $f_{x}(p)=p(x)$ for all $p$ in $C$. Take $Q$ to be the smallest subspace of $Q_{0}$ satisfying $\left(^{*}\right)$ and containing $\left\{f_{x}\right\}_{x \in[0,1]}$.

For $\lambda>0$ denote by $I_{\lambda}$ the transformation from $Q$ to $Q$ such that if $f$ is in $Q$, then

$$
\left(I_{\lambda} f\right)(p)=(1 / \lambda) \int_{0}^{\infty} d u e^{-u / \lambda} f(T(u) p)
$$

for all $p$ in $C$. One can see that $Q$ is composed of $\left\{f_{x}\right\}_{x \in[0,1]}, I_{\lambda_{1}} \cdots I_{\lambda_{n}} f_{x}$ for $\lambda_{1}, \ldots, \lambda_{n}>0, x$ in $[0,1], n=1,2, \ldots$, as well as all finite linear combinations of these elements. If $f$ is in $Q, p$ in $C$ and $\lambda>0$, then $\left(I_{\lambda} f\right)(p)$ is an average (weighted) of $f(T(t) p)$ taken over all $t \geqq 0$. Moreover,

$$
\lim _{\lambda \rightarrow 0}\left(I_{\lambda} f\right)(p)=f(p) \cdot
$$

Now it may be seen that if $f$ is in $Q$ and $\lambda>0$, then $I_{\lambda} f$ is in $D_{A}$ and $A\left(I_{\lambda} f\right)=(1 / \lambda)\left(I_{\lambda} f-f\right)$. Actually, $(I-\lambda A)^{-1}=I_{\lambda}$.

In particular, if $\lambda>0$ and $x$ is in $[0,1]$, then $I_{\lambda} f_{x}$ is in $D_{A}$ and so

$$
\begin{aligned}
& \lim _{t \rightarrow 0}(1 / t)\left[\left(I_{\lambda} f_{x}\right)(T(t) p)-\left(I_{\lambda} f_{x}\right)(p)\right] \\
& \quad=\lim _{t \rightarrow 0}(1 / \lambda) \int_{0}^{\infty} d u e^{-u / \lambda}(1 / t)[(T(t+u) p)(x)-(T(u) p)(x)]=\left(A\left(I_{\lambda} f_{x}\right)\right)(p)
\end{aligned}
$$

exists for all $p$ in $C$. This illustrates a particularly close analogy between the way $A$ is defined and the way one attempts to define a generator $B$. The domain of $B$ is all $p$ in $C$ such that

$$
\lim _{t \rightarrow 0}(1 / t)[T(t) p-p]
$$

exists in $C_{[0,1]}$ (and hence

$$
\lim _{t \rightarrow 0}(1 / t)[(T(t) p)(x)-p(x)]=\lim _{t \rightarrow 0}(1 / t)\left(f_{x}(T(t) p)-f_{x}(p)\right)
$$


exists for all $x$ in $[0,1])$.

In Webb's example, $B$ does not seem adequate for the reconstruction of $T$; from Theorem $8, T$ may be constructed from $A$ by

$$
\lim _{n \rightarrow \infty}\left[(I-(\lambda / n) A)^{-n} f_{x}\right](p)=f_{x}(T(\lambda) p)=(T(\lambda) p)(x)
$$

for all $\lambda \geqq 0, p$ in $C$ and $x$ in $[0,1]$.

7. An example of a quasi-analyticity theorem for a nonlinear semigroup $T$ is Theorem 9. The idea here is to represent $T$ as a linear semigroup on an appropriate space of functions and then apply Theorem 3.

TheOREM 9. Suppose $K=H, T$ is a semigroup on the bounded subset $C$ of $H, Q$ is the smallest subspace of $Q_{0}$ containing $\{T(\lambda)\}_{\lambda \geqq 0}$ and $(S(\lambda) f)(x)$ $=f(T(\lambda) x)$ for all $f$ in $Q, x$ in $C, \lambda \geqq 0$. Then $T$ is quasi-analytic if

$$
\liminf _{\lambda \rightarrow 0}|S(\lambda)-I|<2 \text {. }
$$

Some of the examples shed light on the status of the hypothesis of this theorem.

In Example 4, the space $Q$ may be seen to be dense in the space (using sup norm) of all continuous real-valued functions $f$ on $[-1,1]$ so that $f$ is linear on $[-1,0]$ and $f(0)=0$.

In Example 3, $Q$ is dense in the space (under sup norm) of all continuous functions on $[0,1]$ which are 0 at 0 .

In Example 5, using a theorem of Porcelli [34], $Q$ is dense in the space (under sup norm) of all continuous functions on $[0,1]$ which are 0 at 0 .

In each of these examples, if $S$ is defined as in Theorem 9, it follows that $\lim _{\lambda \rightarrow 0}|S(\lambda)-I|=2$. Roughly, this occurs since the space $Q$ in each instance is so large.

In Example 9, however, $Q$ is all functions on $[-1,1]$ so that $f(0)=0$ and $f$ is linear on both $[-1,0]$ and $[0,1]$. Here $\lim _{\inf _{\lambda \rightarrow 0}}|S(\lambda)-I|<2$.

An interesting problem, perhaps suggested by the above, is that of classifying nonlinear semigroups according to properties of the corresponding minimal space $Q$ which satisfies property (*).

\section{REFERENCES}

1. A. Beurling, On quasi-analyticity and general distributions, Multigraphed Lecture Notes, Stanford University, Stanford, Calif., 1961.

2. - On analytic extension of semigroups of operators, J. Functional Analysis 6 (1970), 387-400.

3. S. Bernstein, Leçons sur les propriétés extrémales et la meilleure approximation des fonctions analytiques d' une variable réelle, Gauthier-Villars, Paris, 1926.

4. H. Brezis and A. Pazy, Semigroups of nonlinear contractions on convex sets, J. Functional Analysis 6 (1970), 237-281.

5. P. L. Butzer and H. Berens, Semi-groups of operators and approximation, Die Grundlehren der math. Wissenschaften, Band 145, Springer-Verlag, New York, 1967. MR37 \# 5588 . 
6. T. Carleman, Les fonctions quasi-analytiques, Gauthier-Villars, Paris, 1926.

7. M. Crandall and T. Liggett, $A$ theorem and a counterexample in the theory of semigroups of nonlinear transformations, Trans. Amer. Math. Soc. 160 (1971), 263-278.

8. - Generation of semi-groups of nonlinear transformations on general Banach spaces, Amer. J. Math. 93 (1971), 265-298.

9. J. R. Cuthbert, On semigroups such that $T_{t}-I$ is compact for some $t>0, \mathrm{Z}$. Wahrscheinlichkeitstheorie und Verw. Gebiete 18 (1971), 9-16.

10. J. R. Dorroh, A nonlinear Hille-Yosida-Phillips theorem, J. Functional Analysis 3 (1969), 345-353. MR39 \# 2019.

11. E. Hille and R. S. Phillips, Functional analysis and semi-groups, rev. ed., Amer. Math.

Soc. Colloq. Publ., vol. 31, Amer. Math. Soc., Providence, R.I., 1957. MR19, 664.

12. E. L. Ince, Ordinary differential equations, Dover, New York, 1944. MR6, 65.

13. T. Kato, Note on the differentiability of nonlinear semigroups, Proc. Sympos. Pure

Math., vol. 16, Amer. Math. Soc., Providence, R.I., 1970, pp. 91-94. MR42 \# 5100.

14. - A characterization of holomorphic semigroups, Proc. Amer. Math. Soc. 25 (1970), 495-498. MR41 \#9050.

15. D. G. Kendall, Some recent developments in the theory of denumerable Markov processes, Trans. Fourth Prague Conference on Information Theory, Statistical Decision Functions, Random Processes (Prague, 1965), Academia, Prague, 1967, pp. 11-27. MR36 \#974.

16. Y. Komura, Differentiability of nonlinear semigroups, J. Math. Soc. Japan 21 (1969), 375-402. MR40 \# 3358 .

17. N. Levinson, Gap and density theorems, Amer. Math. Soc. Colloq. Publ., vol. 26, Amer. Math. Soc., Providence, R.I., 1940. MR2, 180.

18. S. Mandelbrojt, Séries de Fourier et classes quasi-analytiques de fonctions, GauthierVillars, Paris, 1935.

19. - Séries adhérentes, régularisation des suites, applications, Gauthier-Villars, Paris, 1952. MR 14, 542.

20. I. Miyadera, Some remarks on semigroups of nonlinear operators, Tôhoku Math. J. (2) 23 (1971), 245-258.

21. J. W. Neuberger, A quasi-analyticity condition in terms of finite differences, Proc. London Math. Soc. (3) 14 (1964), 245-259. MR 28 \# 3130.

22. - Quasi-analyticity of trajectories of semigroups of bounded linear transformations, Notices Amer. Math. Soc. 12 (1965), 815. Abstract \# 65T-454.

23. , An exponential formula for one-parameter semi-groups on nonlinear trans-

formations, J. Math. Soc. Japan 18 (1966), 154-157. MR 34 \# 622.

24. - Quasi-analytic collections containing Fourier series which are not infinitely

differentiable, J. London Math. Soc. 43 (1968), 612-616. MR 37 \# 5344.

25.

Math. Soc. 25 (1970), 488-494. MR 41 \# 4296.

26. - Quasi-analyticity and semigroups of bounded linear transformations, Bull.

Amer. Math. Soc. 78 (1972), 85-87.

27. ㄴ, Lie generators for strongly continuous equi-continuous one-parameter semigroups on a metric space, Indiana Univ. Math. J. 21 (1972), 961-971.

28. - Lie generators for one-parameter semigroups of transformations, J. Reine Angew. Math. (to appear).

29. - Quasi-analytic semigroups of bounded linear transformations, J. London Math. Soc. (to appear).

30. S. Oharu, Note on the representation of semi-groups of non-linear operators, Proc. Japan Acad. 42 (1966), 1149-1154. MR 36 \# 3167.

31. J. M. Page, Ordinary differential equations, Macmillan, New York, 1897.

32. A. Pazy, Approximations of the identity operator by semigroups of linear operators, Proc. Amer. Math. Soc. 30 (1971), 147-150.

33. R. E. A. C. Paley and N. Wiener, Fourier transforms in the complex domain, Amer. Math. Soc. Colloq. Publ., vol. 19, Amer. Math. Soc., Providence, R.I., 1934.

34. P. Porcelli, Uniform completeness of sets of reciprocals of linear functions, Duke Math. J. 20 (1953), 185-193. MR 15, 107.

35. D. Rutledge, A generator for a semigroup of nonlinear transformations, Proc. Amer. Math. Soc. 20 (1969), 491-498. MR 39 \# 821.

36. G. Sansone, Orthogonal functions, Zanichelli, Bologna, 1952; English transl., Pure and Appl. Math., vol. 9, Interscience, New York, 1959. MR 13, 741; MR 21 \# 2140.

37. A. F. Timan, Theory of approximation of functions of a real variable, Fizmatgiz, Moscow, 1960; English transl., Internat. Series of Monographs in Pure and Appl. Math., vol. 34, Macmillan, New York, 1963. MR 33 \# 465. 
38. Ch. de la Vallée Poussin, Quatre leçons sur les fonctions quasi-analytiques de variable réelle, Bull. Soc. Math. France 52 (1924), 175-203.

39. G. F. Webb, Nonlinear contraction semigroups in weakly complete Banach space, Thesis, Emory University, Atlanta, Ga., 1968.

40. - Representation of semi-groups of nonlinear nonexpansive transformations in Banach spaces, J. Math. Mech. 19 (1969/70), 159-170. MR 40 \# 793.

41. D. Williams, On operator semigroups and Markov groups, Z. Wahrscheinlichkeitstheorie und Verw. Gebiete 13 (1969), 280-285. MR 40 \# 8127.

42. K. Yosida, Functional analysis, Die Grundlehren der math. Wissenschaften, Band 123, Academic Press, New York; Springer-Verlag, Berlin, 1965. MR 31 \# 5054.

Department of Mathematics, Emory University, Atlanta, Georgia 30322 